\title{
A behavioral analysis of spatial neglect and its recovery after stroke
}

\author{
Jennifer Rengachary ${ }^{1 *}$, Biyu J. He ${ }^{2}$, Gordon L. Shulman ${ }^{1}$ and Maurizio Corbetta ${ }^{1,2,3 *}$ \\ 1 Department of Neurology, Washington University School of Medicine, St. Louis, MO, USA \\ 2 Department of Radiology, Washington University School of Medicine, St. Louis, MO, USA \\ ${ }^{3}$ Department of Anatomy and Neurobiology, Washington University School of Medicine, St. Louis, MO, USA
}

Edited by:

Donald T. Stuss, Baycrest Centre for Geriatric Care, Canada

Reviewed by:

Joseph M. Degutis, Harvard University, USA

Tom Manly, University of Cambridge,

UK

*Correspondence:

Jennifer Rengachary and Maurizio

Corbetta, Department of Neurology,

Washington University School of

Medicine, Box 8225, 4525 Scott

Avenue, St. Louis, MO 63110, USA.

e-mail: jstruna@npg.wustl.edu;

mau@npg.wustl.edu
In a longitudinal study of recovery of left neglect following stroke using reaction time computerized assessment, we find that lateralized spatial deficits of attention and perception to be more severe than disturbance of action. Perceptual-attention deficits also show the most variability in the course of recovery, making them prime candidates for intervention. In an anatomical analysis of MRI findings, ventral frontal cortex damage was correlated with the most severe neglect, reflecting impaired fronto-parietal communication.

Keywords: stroke, hemispatial neglect

\section{INTRODUCTION}

Unilateral spatial neglect (or neglect) is one of the most frequent disorders following stroke, involving about $25-30 \%$ of all patients, an estimated 250,000 patients per year in the US (Pedersen et al., 1997; Appelros et al., 2002; Buxbaum et al., 2004). It occurs for lesions to either hemisphere, but it is more severe and enduring after right hemisphere damage (Stone et al., 1993). Although neglect tends to improve spontaneously, it is associated with poor motor recovery, higher disability, and poor response to rehabilitation (Katz et al., 1999; Cherney et al., 2001; Paolucci et al., 2001; Buxbaum et al., 2004).

The hallmark of neglect is a difficulty in attending and responding to stimuli on the side of space or the body opposite to the lesion (Heilman et al., 1987; Mesulam, 1999; Halligan et al., 2003). Although it is well accepted that the complex behavioral syndrome of neglect involves a constellation of deficits (Barrett et al., 2006), it is not at all clear which deficits are the most relevant clinically in terms of frequency, severity, and change over time in relation to recovery. One component of neglect is a lateralized perceptual impairment modulated by spatial attention (Posner et al., 1984; Morrow and Ratcliff, 1988; Friedrich et al., 1998) and by the position of sensory stimuli in relation to the body (Behrmann and Tipper, 1999). Another hypothesized element of neglect is a lateralized deficit of action in which patients are delayed in initiating movements to the contralesional space, termed directional hypokinesia (Heilman et al., 1985; Mattingley et al., 1998; Husain et al., 2000; Sapir et al., 2007). It is unclear how common this deficit of action is, or how its evolution relates to that of perceptual impairments. To our knowledge these deficits have not been studied jointly in a large longitudinal group of neglect patients. Here, we test the hypothesis that the two types of deficits may show different recovery trajectories.
Lateralized spatial perceptual deficits were investigated using the Posner task, developed by Posner (1980), in which subjects detect visual stimuli at attended or unattended peripheral locations while maintaining central fixation. This task has been used widely in studies of left neglect as it is sensitive to neglect even in mild cases (Morrow and Ratcliff, 1988; Friedrich et al., 1998; Bartolomeo and Chokron, 2002; Rastelli et al., 2008). A relative response time (RT) delay or lower accuracy for targets presented in the contralesional, as compared to the ipsilesional, visual field indexes a lateralized deficit in visual perception and attention (typically in the left visual field after right hemisphere stroke). A relative delay in responding to targets at unattended, as compared to attended locations, indexes a deficit of reorienting spatial attention. A third deficit concerns difficulties in responding to unattended targets in the contralesional field, the "disengagement" deficit, reflecting a deficit in disengaging attention from the ipsilesional field. These indices are abnormal in patients with extinction (a milder form of neglect), with studies particularly emphasizing the disengagement effect (Posner et al., 1984; Morrow and Ratcliff, 1988; Friedrich et al., 1998), which correlates with clinical measures of neglect recovery (Morrow and Ratcliff, 1988).

Lateralized deficits of action were measured using the Reach task, based on a test developed by Mattingley et al. (1998), in which subjects reach toward a stimulus presented in the periphery of the visual field. This task was chosen as it elegantly distinguishes visual-perceptual from directional motor deficits. Perceptual deficits are assessed by a difference in RT between targets presented in the visual field opposite (contralesional) or on the same side of the lesion (ipsilesional). Directional motor deficits are assessed by a difference in RT for reaching in a contralesional direction (leftward movements in the case of right hemisphere damage) or an 
ipsilesional direction, independent of target position. Directional hypokinesia is assessed by a difference in RT for reaches in a contralesional or ipsilesional direction specifically for contralesional targets: consider a situation in which subjects reach to a left visual field target from a midline position (center start) using a leftward trajectory. For a subject with left neglect, perceptual, and motor neglect are coincident in this condition. Consider, however, a situation in which the starting hand position is to the left of the left target position. When a left target is presented, the position of the stimulus still falls in the contralesional visual field, but now subjects must perform a rightward reach to touch it. In this case, perceptual and motor directions are dissociated.

A final goal was to use anatomical analysis to investigate whether damage to brain regions, commonly lesioned in neglect that partially overlap regions involved in spatial orienting (dorsal attention network) and reorienting (dorsal and ventral attention networks; Corbetta and Shulman, 2002; Corbetta et al., 2008), produces a differential effect on lateralized orienting and lateralized action. In light of recent findings suggesting a key role for the ventral frontal cortex (VFC) in mediating interactions between the dorsal and ventral attention networks (He et al., 2007; Asplund et al., 2010), we tested the hypothesis that lesions to VFC produce neglect.

\section{MATERIALS AND METHODS SUBJECTS}

Sixty-one patients were enrolled prospectively after providing informed consent in agreement with procedures established by the Washington University Institutional Research Board. Our patients represent a consecutive sample of qualifying patients with a clinical diagnosis of neglect admitted to the Rehabilitation Institute of St. Louis. Thirty patients were tested longitudinally: first at the acute stage [mean time: $15 \pm 6(1 \mathrm{SD})$ days after stroke onset], then at the chronic stage (mean time: $35 \pm 5$ weeks after stroke onset). Because these subjects were enrolled as part of a larger functional imaging study, they were not tested or scanned prior to 10 days post-stroke, to allow time for autoregulation to normalize. The chronic timepoint was chosen to allow recovery of neglect symptoms to plateau while minimizing attrition from the study. The patients who were unable to return for a chronic visit did not differ significantly from those tested longitudinally in terms of demographics, acute neglect severity, or overall stroke severity. We also tested a group $(N=30)$ of age-matched controls (mean age $62.7 \pm 15.1$ ). Demographics for the longitudinal group are shown in Table 1.

\section{Inc/usion/exc/usion criteria for neglect subjects}

Inclusion criteria: (1) Age $\geq 18$. (2) Single right hemisphere stroke, ischemic or hemorrhagic. (3) Clinical evidence of neglect (see Clinical Tasks for the Diagnosis of Neglect). (4) Alert and capable participating in research.

Exclusion criteria: (1) Evidence by CT or MRI of other strokes, although up to two lacunes were allowed in the subcortical white matter. (2) Presence of other neurological, psychiatric, or medical conditions that altered the interpretation of the behavioral/ imaging studies (e.g., dementia), or life expectancy less than 1 year (e.g., cancer). (3) Abnormal score (>13) on the Short Blessed Scale (Katzman et al., 1983), to rule out patients with dementia. (4) Homonymous hemianopsia.
Of all patients admitted with right hemisphere acute stroke at the hospital during the course of study enrollment, $30 \%$ had a diagnosis of left neglect in their clinical chart. Of these patients, $16 \%$ were enrolled. Of those not enrolled, $30 \%$ were excluded due to evidence of other lesions (prior history of stroke, radiological evidence of old infarcts); $35 \%$ due to comorbid medical history (prior brain injury, dementia, life expectancy less than 1 year, etc.); $26 \%$ due to homonymous hemianopsia; $8 \%$ due to contraindication for MRI (pacemaker, etc.); $1 \%$ due to low level of consciousness; and $8 \%$ refused. Subjects were excluded for low level of consciousness only if they were completely unable to follow directions to participate in the study tasks; those who were drowsy but arousable were allowed rest breaks as needed.

\section{Clinical tasks for the diagnosis of neglect}

Patients were included on the basis of at least one positive test of neglect. All acute patients were tested on at least five of the six tests below.

1. Extinction: A test of double simultaneous stimulation in visual, auditory, and tactile modalities. For visual, the tester wiggles two fingers at $10^{\circ}$ eccentricity. For tactile, blindfolded subjects are touched on the dorsal surface of the second finger. For auditory, the experimenter rubs his/her fingers near the subject's ear(s). Subjects report detection of the stimulus on left, right, or both sides. The test is not performed in the presence of primary sensory deficits. (Cut off: miss $>1$ out 6 contralesional stimuli in any modality).

2. Behavioral inattention test (BIT) star (Wilson et al., 1987; Halligan et al., 1991). A visuospatial scanning test with an array of stars and letters on a paper. (Cut off $<51$ targets of 54 canceled).

3. Mesulam shape cancelation (Mesulam, 1985). A more difficult visuospatial scanning test with a cluttered array of symbols. (Cut off $<56$ targets of 60 canceled).

4. Catherine Bergego Scale (Azouvi et al., 2003). A measure of neglect in activities of daily living. The treating occupational therapist reports difficulties in dressing, eating, and environmental navigation due to neglect. (Cut off score $>1$ of 30 ). This test was applied only at the acute stage as most participants were discharged from therapy by the chronic visit.

5. The baking tray test (Tham and Tegner, 1996). A functional measure of neglect. Patients arrange "cookies" on a tray, attempting to distribute evenly from right to left. Number of cookies displaced is counted. (Cut off $<24$ of 32 cookies placed correctly).

6. Behavioral inattention test reading (Wilson et al., 1987; Halligan et al., 1991). A functional measure of neglect. Patients read aloud an article in three columns (left, center, right). (Cut off $>8$ of 153 words omitted).

\section{APPARATUS AND STIMULI}

Subjects were tested in dim, quiet testing rooms. Stimuli were generated by an Apple Power Macintosh computer and projected onto a 17-inch Apple Monitor. Behavioral responses were acquired through a Carnegie Mellon button box interfaced with the computer. The head was stabilized with a chin rest. The experimenter visually screened eye movements and encouraged visual fixation when a breakdown of 
Table 1 | Characteristics of longitudinal stroke subjects.

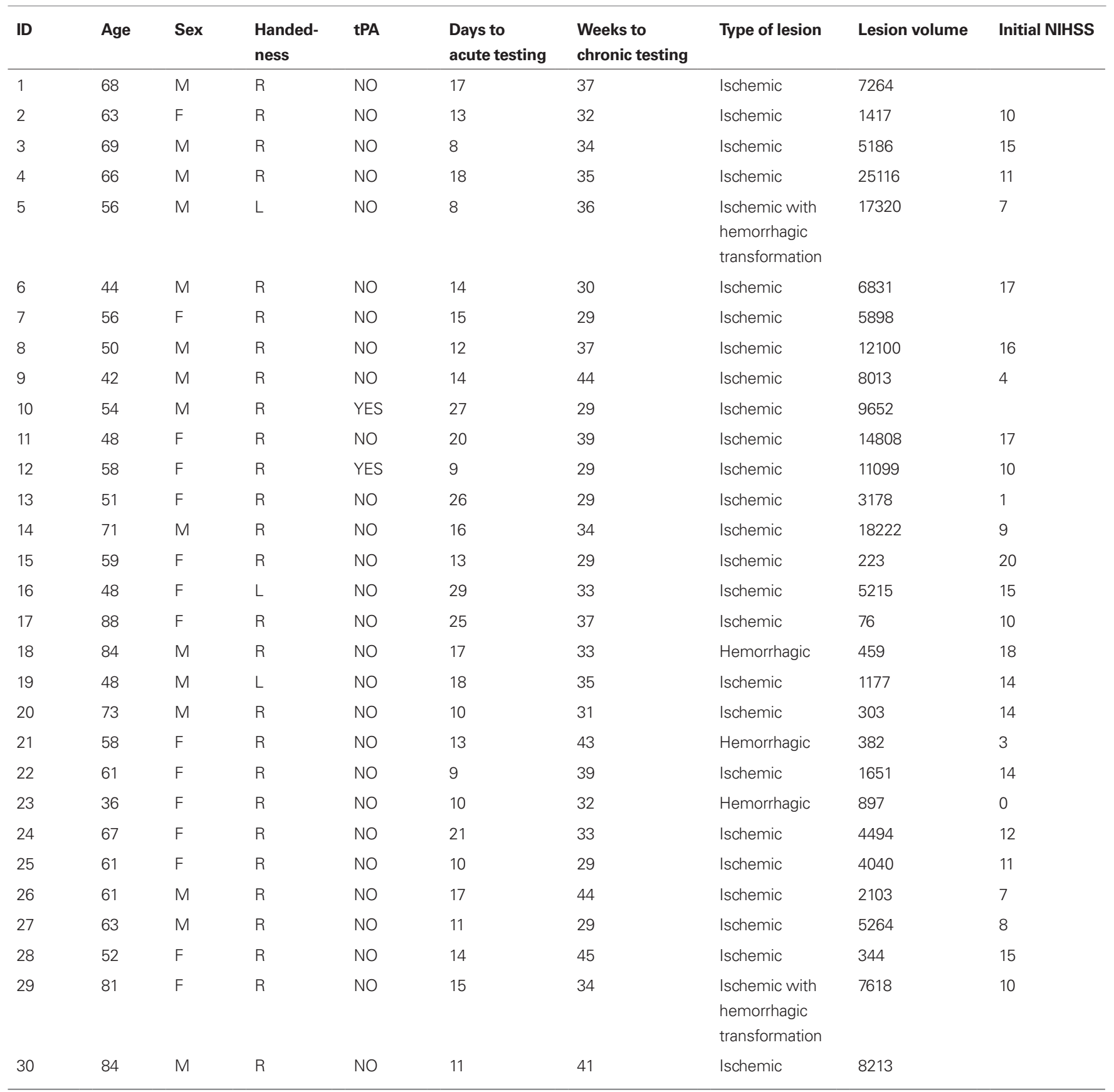

Age: age at stroke onset; tPA: tissue plasminogen activator administered in Emergency Room at stroke onset; type of lesion: ischemic or hemorrhagic stroke; lesion volume: voxels defined as infarcted area in lesion segmentation; initial NIHSS: score on National Institutes of Health Stroke Scale administered approximately 24-72 h after stroke onset, in which $0=$ normal, mild = 1-7, moderate $=8-16$, and severe $>16$.

fixation occurred. In the case of a quadrantanopsia, the stimuli were presented in the visible part of the field on symmetrical opposite positions across the vertical meridian. Five patients had quadrantanopsia.

\section{PROCEDURES}

Posner task (Posner, 1980)

The display contained two square frames positioned along the horizontal meridian to the left and right of a fixation point (center of frame $3.3^{\circ}$ from fixation point). Each trial started with the fixation point changing from red to green. After $800 \mathrm{~ms}$, an arrow cue pointing left or right was presented at fixation for $2360 \mathrm{~ms}$. Following a delay ranging from 1000 to $2200 \mathrm{~ms}$ the target (an asterisk) was presented for $300 \mathrm{~ms}$ within one of the two frames (left, right). On $75 \%$ of the trials, the target was presented at the location indicated by the cue, while on $25 \%$ of the trials it was presented at the opposite location. The subject detected the target as quickly as possible with a dominant hand key-press, and RT and accuracy were measured. Each trial was 
separated by an inter-trial interval (ITI) of $2360 \mathrm{~ms}$. Each block contained 40 trials, and two blocks were run in each subject. There were no catch trials.

\section{Reach task (Mattingley et al., 1998)}

The display contained a fixation point. Each trial started with the subject pressing a key with the dominant index finger. After a random interval (1500-3000 ms), the target (an asterisk) was presented for $2000 \mathrm{~ms}$ at a left or right location along the horizontal meridian ( $10^{\circ}$ from fixation point). We used long target durations to minimize problems with working memory (i.e., pointing to remembered target locations). The subject released the key as fast as possible and touched the target location on the screen. After returning the finger to the keypad, the next trial began following a fixed interval of $500 \mathrm{~ms}$. Three conditions were run to dissociate the position of the target and the direction of reach: (a) central starting position (button box positioned at midline); (b) left starting position (button box positioned about $20^{\circ}$ to the left of fixation, a relative left position of about $10^{\circ}$ with respect to the left target location); (c) right starting position. Each block contained 30 trials (15 left, 15 right). One block for each starting position was run in each subject.

\section{ANATOMY}

\section{Lesion segmentation in stroke patients}

Structural scans including a T1-weighted (T1W) sagittal magnetization-prepared rapid gradient echo (MPRAGE; TR $=1900 \mathrm{~ms}$, $\mathrm{TI}=1100 \mathrm{~ms}, \mathrm{TE}=3.9 \mathrm{~ms}$, flip angle $15^{\circ}, 1 \mathrm{~mm} \times 1 \mathrm{~mm} \times 1.25 \mathrm{~mm}$ voxels) and a T2-weighted (T2W) fast spin echo scan (TR $=4380 \mathrm{~ms}$, $\mathrm{TE}=94 \mathrm{~ms}, 1 \mathrm{~mm} \times 1 \mathrm{~mm} \times 3 \mathrm{~mm}$ ) were obtained at the chronic stage. T1-weighted and T2-weighted structural magnetic resonance images were segmented into regions corresponding to gray matter, white matter, cerebrospinal fluid, and infarct based on a bispectral fuzzy class means semi-automated procedure after gainfield correction as previously described (Sheline et al., 2008). The total number of infarcted voxels in each slice was calculated using Analyze AVW (The Biomedical Imaging Resource at the Mayo Foundation) and binary maps representing lesioned space were produced and transformed into atlas space.

\section{Division into anatomical subgroups}

Stroke patients were subdivided into different anatomical subgroups using a clustering algorithm based on the percentage of overlap between each lesion and a predefined set of anatomical templates [temporo-parietal junction (TPJ), VFC, frontal eye field (FEF), intraparietal sulcus (IPS)] derived from a meta-analysis of functional magnetic resonance imaging (fMRI) studies of attention in normal subjects (He et al., 2007). There were four target cortical templates (IPS, FEF, VFC, TPJ that includes separate regions in supramarginal and superior temporal gyri), all in the right hemisphere, which corresponded to the core regions of the dorsal and ventral fronto-parietal attention networks (Corbetta and Shulman, 2002). The templates were created by a fixed effect analysis in which significant statistical images (i.e., multiple corrected over the whole brain) from multiple experiments were summed and divided by the square root of the number of experiments. The dorsal templates (IPS, FEF) corresponded to regions found to be active across multiple experiments when subjects covertly directed spatial attention to a peripheral visual location after a centrally located cue. The ventral templates (VFC, TPJ) corresponded to regions that are more active when subjects detect targets at unattended as compared to attended locations as in the Posner task (Corbetta et al., 2000; Astafiev et al., 2003; Kincade et al., 2005).

\section{RESULTS}

\section{ANATOMICAL ANALYSIS}

Figure 1 shows the lesion anatomy of the 30 subjects with spatial neglect according to the inclusion criteria who returned at the chronic stage, at which point anatomical images were obtained. The most consistent lesion ( $\sim 65 \%$ of sample or about 20 out 30 subjects) includes the corona radiata just lateral to the ventricle in the frontal white matter. Other regions with $>20 \%$ damage (6-8 out 30 subjects) include the inferior and middle frontal gyrus, superior temporal gyrus (STG), inferior parietal lobule, insula, putamen, caudate, and frontal-temporal-parietal white matter.

\section{NEUROPSYCHOLOGICAL TESTS}

Figure 2 shows the results for the neuropsychological tests used for inclusion. Control subjects scored at or near ceiling on all tests, with fewer than $0.1 \%$ misses on average for each test. Mean number of misses was above cut off for diagnosis with neglect for each of the tasks at the acute stage. Performance on all tasks improved from acute to chronic, but most tests still remained abnormal at the chronic stage.

\section{WHOLE GROUP ANALYSIS Posner task}

The Posner task measures the ability to attend to and detect peripheral visual targets and reorient toward unexpected targets. We first examined the sensitivity of the Posner task in detecting deficits at acute and chronic stages separately, as compared to a group of healthy age-matched controls. An ANOVA was conducted with Group (e.g., Acute, Controls), Visual Field (Left, Right), and Validity (Invalid Targets, Valid Targets) as factors. Because patients missed many trials, including several patients who missed all trials in the left invalid condition (Figure 3A), the longest possible RT (2000 ms) was substituted for missed trials in order to create a unified index that took into account both accuracy and speed (Figure 3B).

A lateralized bias in visual perception, as indicated by the interaction of Visual Field (Left, Right) $\times$ Group (Neglect, Controls), was strongly present at both the acute and chronic stages (Acute, $F_{1,54}=75.476, p<0.001$; Chronic, $F_{1,54}=16.048, p<0.001$ ). Patients were also impaired as compared to controls in responding to unattended targets (Validity $\times$ Group: Acute vs. Control, $F_{1,54}=20.457$, $p<0.001$; Chronic vs. Control, $\left.F_{1,54}=10.197, p=0.002\right)$. The disengagement effect, reflecting poorer performance for unattended targets in the left visual field, was significantly larger in acute patients than controls (Visual Field $\times$ Validity $\times$ Group: $F_{1,54}=9.628$, $p=0.003)$ but not in chronic patients $\left(F_{1,54}=2.809, p=0.099\right)$. While acute patients showed a significantly larger disengagement effect than controls, however, they also showed a significantly larger deficit in responding to unattended targets in the right, ipsilesional field (Group $\times$ Validity, Acute vs. Control: $F_{1,56}=8.50, p=0.005$ ). This latter result indicates that patients showed a reorienting deficit that extended throughout the visual field. 

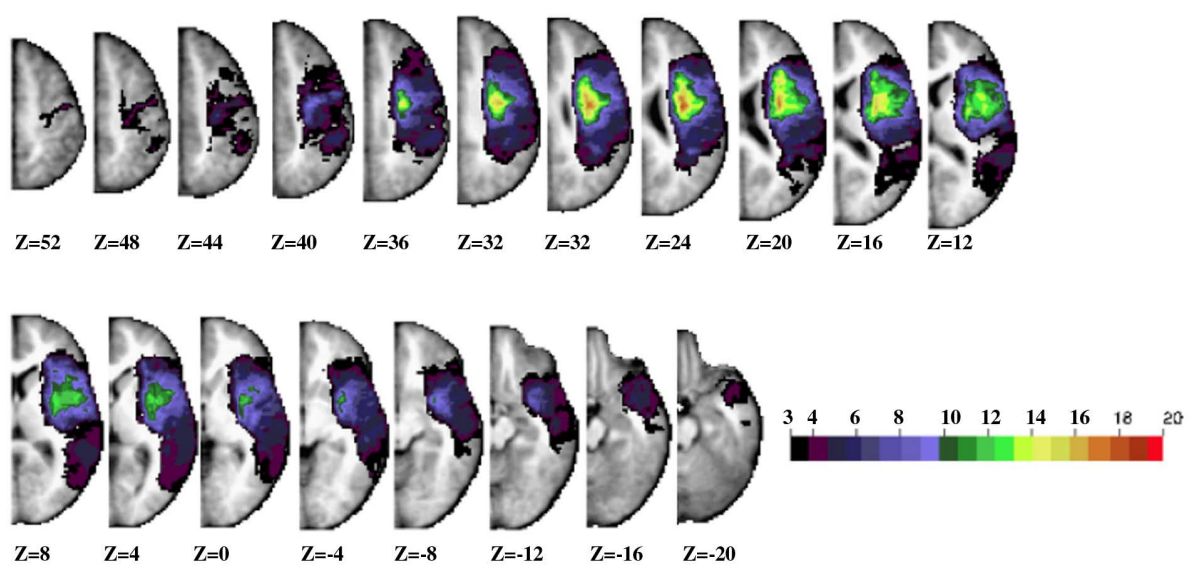

FIGURE 1 | Lesion anatomy. Horizontal slices of anatomical MRI standardized in Talairach atlas showing the lesion distribution for the longitudinal 30 patients. The color scale represents the number of patients with damage in a specific voxel.

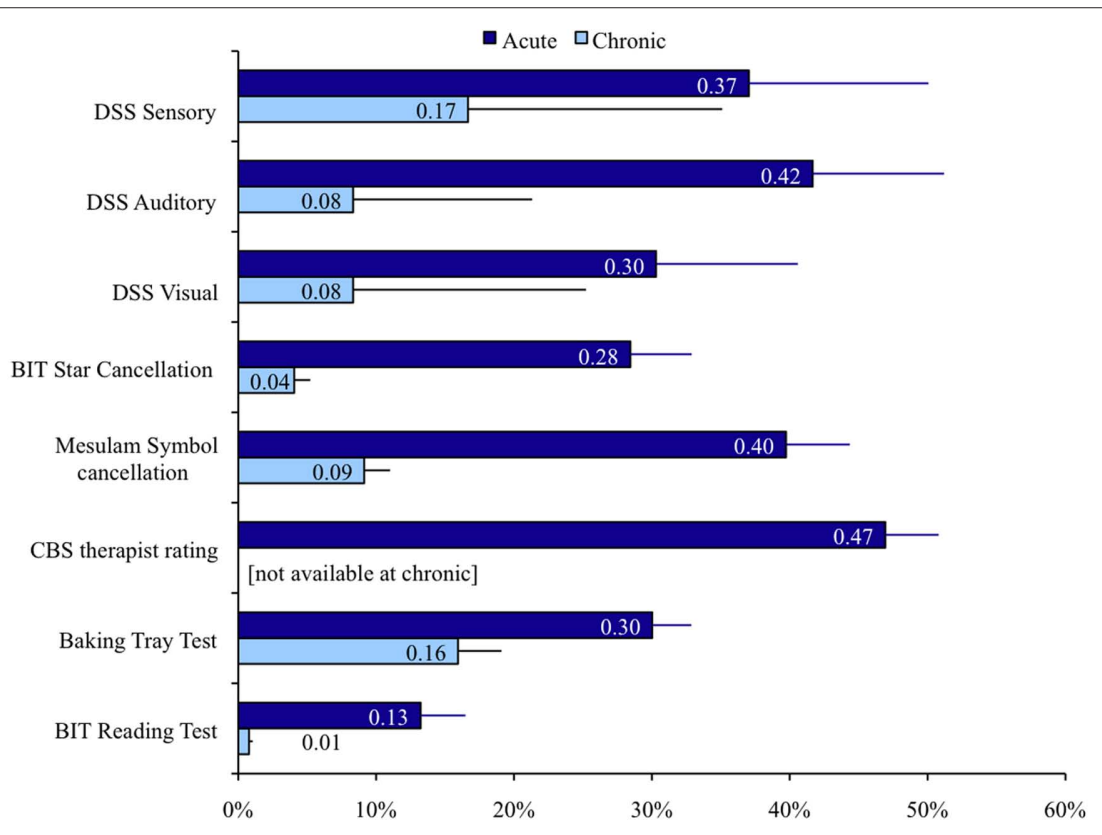

FIGURE 2 | Performance on clinical neglect tests. Percent of targets missed and SEM is displayed for each test. DSS: left side stimuli missed in double simultaneous stimulation. BIT star cancelation: missed targets; Mesulam symbol cancelation: missed targets; CBS therapist rating: severity of neglect in ADL as scored by therapist; baking tray test: incorrectly placed "cookies"; BIT reading test: Words omitted.

We next examined whether deficits in detection, reorienting, and disengagement improved over time, via an ANOVA with Stage (Acute, Chronic), Visual Field (Left, Right), and Validity (Valid Targets, Invalid Targets) as factors. Patients performed worse in the left than right visual fields (Visual Field, $\left.F_{1,25}=72.759, p<0.001\right)$ and in the unattended vs. attended fields (Validity, $F_{1,25}=17.414, p<0.001$ ). A lateralized reorienting bias, or disengagement effect, as measured by the interaction of VF $\times$ Validity, was also present $\left(F_{1,25}=5.771, p=0.024\right)$. All three deficits significantly improved over time (Stage $\times$ VF, $F_{1,25}=28.051, p<0.001 ;$ Stage $\times$ Validity, $F_{1,25}=8.488, p=0.007$; Stage $\times$ VF $\times$ Validity, $\left.F_{1,25}=8.244, p=0.008\right)$.
Overall, this sample of neglect patients showed severe deficits in detection and reorienting that were particularly marked in the contralesional visual field. These impairments significantly improved over time although most were still present at the chronic stage post-injury.

\section{Reach task}

The Reach task dissociates lateralized visual detection from lateralized action. As no patient missed every trial for a given condition, RT and accuracy were computed separately (Figure 4).

When neglect patients were compared to healthy controls at each stage separately, the lateralized bias in perception, as indexed by the interaction of Visual Field (Left, Right) $\times$ Group (Neglect, 

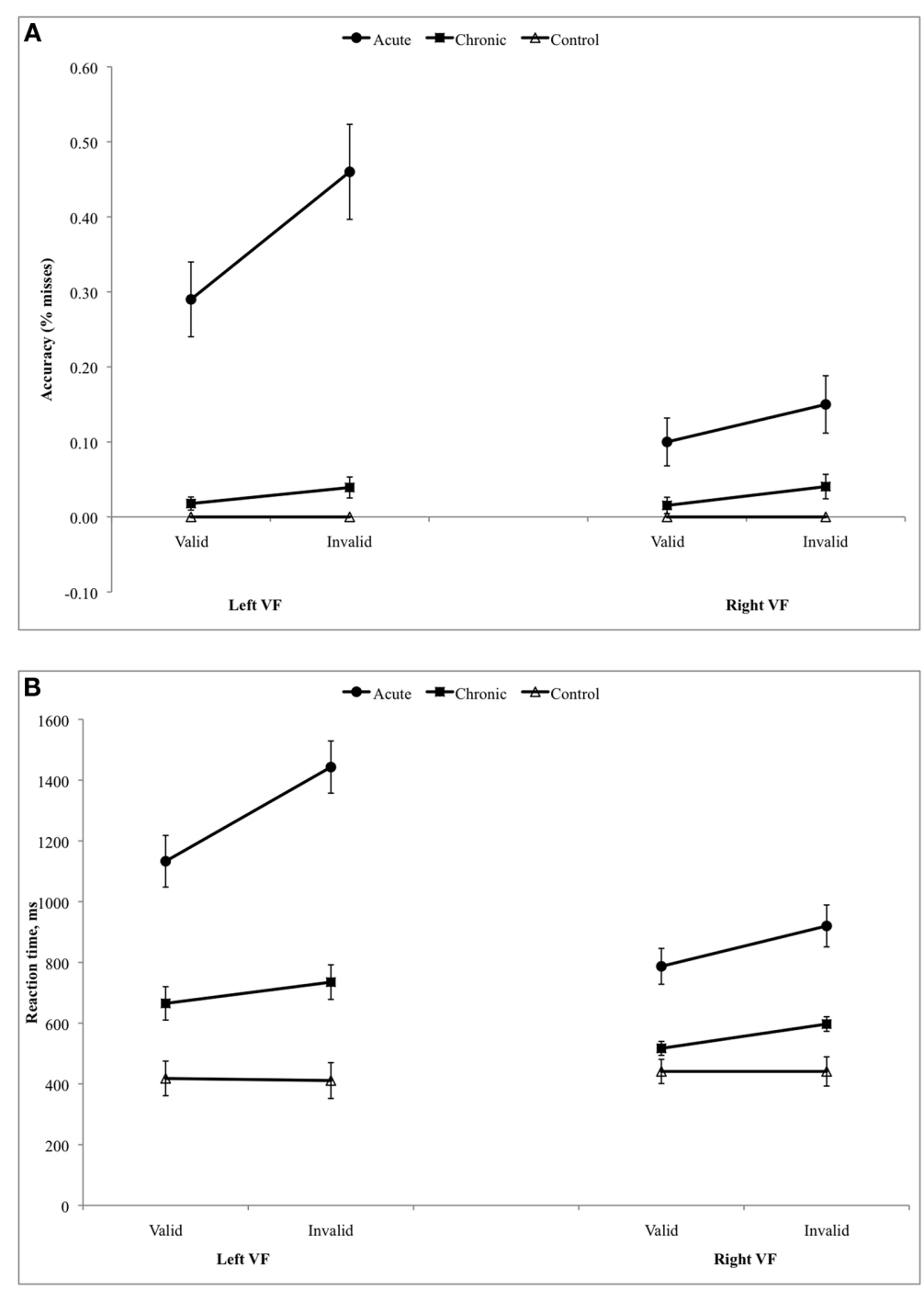

FIGURE 3 | (A) Results of Posner task, accuracy. (B) Results of Posner task, reaction time (substituting 2 s for miss trials).

Controls), was strongly significant at both the acute and chronic stages (RT: Acute, $F_{1,56}=13.958, p=0.001$; Chronic, $F_{1,56}=4.228$, $p=0.041 ; \%$ Misses: Acute, $F_{1,56}=15.627, p<0.001$; Chronic $\left.F_{1,56}=6.516, p=0.011\right)$. In contrast, no difference in movement direction was detected between neglect and controls [Movement (Left move, Right move) $\times$ Group (Neglect, Controls): (RT: Acute, $F_{1,56}=0.094$, n.s.; Chronic, $F_{1,56}=0.160$, n.s.], nor was there any evidence of directional hypokinesia as there was no significant interaction of Group $\times$ Movement $\times$ VF (RT: Acute, $F_{1,56}=0.191$, n.s.; Chronic, $F_{1,56}=0.189$, n.s.).

We next compared acute and chronic patients in order to assess recovery from neglect. The patients' perceptual deficit in the left visual field (RT: Left vs. Right VF: 738 vs. $607 \mathrm{~ms}$, Visual Field, $F_{1,27}=53.643, p<0.001$; \% Misses: Left vs. Right: 7.3 vs. $0.5 \%$. VF, $\left.F_{1,27}=13.962, p=0.001\right)$ significantly improved over time (Stage $\times$ VF: RT, $F_{1,27}=6.519, p=0.017$; \% Misses: $\left.F_{1,27}=4.827, p=0.037\right)$. In contrast, there was only a mild deficit for reaching to the left (Left vs. Right move: 678 vs. $668 \mathrm{~ms}$,
Movement, $F_{1,27}=0.045, p=$ n.s.; \% Misses: Left vs. Right move $F_{1,27}=.045, p=$ n.s.), which did not change from acute to chronic stages (Stage $\times$ Movement: RT, $F_{1,27}=0.289, p=$ n.s.; \% misses, $F_{1,27}=3.190, p=$ n.s.; Figure 4). There was also no evidence of directional hypokinesia, given the lack of any significant interaction of Movement $\times \mathrm{VF}$.

\section{Relationship between acute performance and chronic recovery}

In general, acute performance on the Posner task better predicted recovery on the battery of clinical neglect tests than did performance on the Reach task. Chronic subjects were classified as "recovered" if they showed neglect on at most one of the administered clinical tests (according to published norms) and as "did not recover" if they showed neglect on two or more tests (26/30 of the acute patients had shown deficits on at least $4 / 5$ or $4 / 6$ of the administered tests).

While the acute movement direction effect on the Reach task was higher in the "not recovered" group (108 vs. $19 \mathrm{~ms}$ ), this difference was not significant $\left(F_{1,25}=2.822\right.$, n.s. $)$. Directional hypokinesia was 


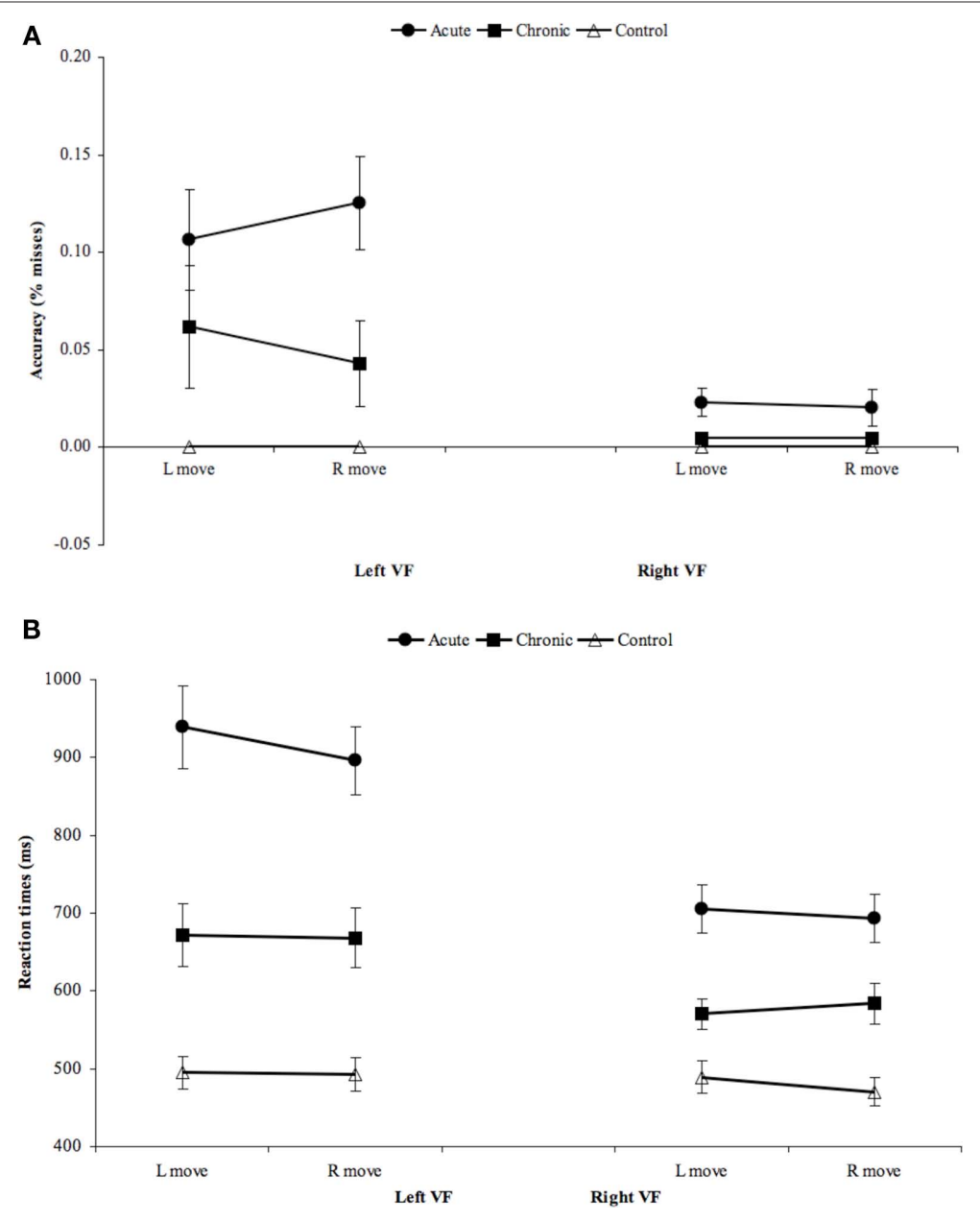

FIGURE 4 | Results of Reach task. (A) Reaction time, (B) Accuracy.

absent in both groups at acute and chronic stages, and no directional motor effects were present in either group at the chronic stage (Figure 6).

For the Posner task, the visual field effect was significantly higher at the acute stage for subjects who "did not recover" (635 vs. $306 \mathrm{~ms}$, $\left.F_{1,21}=8.462, p=0.008\right)$ but not the disengagement or validity effects, or the average RT in the ipsilesional field. However, as shown in the scatter plot of scores for each patient at the acute stage, even the visual field effect scores did not allow a good prediction of whether an individual would recover by the chronic stage.

As seen in Figure 5D, there was little correlation between improvement from acute to chronic testing in the Posner and Reach tasks.

In summary, this analysis confirms that a strong perceptual bias was present at the acute stage but lessened over time; in contrast, directional motor deficits were neither prominent nor significantly changed over time.

\section{ANATOMICAL SUBGROUP ANALYSIS}

Using the predefined templates, patients were found to belong to one of three main subgroups: subcortical $(N=13)$, VFC/Insular (referred to as VFC henceforth; $N=9$ ), and TPJ $(N=4)$, or two minor subgroups: FEF $(N=2)$, Occipital $(N=2)$. The latter two groups were excluded from further analysis, as was one VFC patient who did not complete the computerized testing. No IPS patient was identified. Both the subcortical and the occipital subgroups had less than $10 \%$ overlap with any of the four templates, but these two groups were easily separated based on their lesion location.

In the subcortical group the average lesion involved predominantly the right putamen $(-50-60 \%$ overlap), and the frontal and sub-insular white matter ( $-30 \%$ overlap; Figure 5$)$. In the VFC group the most common site of injury involved the inferior frontal gyrus, frontal operculum, insula, and corona radiata (>90\% overlap; Figure 7A). Other regions of damage included the precentral gyrus, parietal operculum, and postcentral cortex $(\sim 40-70 \%$ overlap), and the anterior supramarginal gyrus and internal capsule $(-30 \%$ overlap). Finally in the TPJ group, the core damage involved the middle and posterior portion of the STG (>90\% overlap; Figure 7B). Other regions included the supramarginal and angular gyri and underlying white matter, parietal operculum, and insula ( $70-90 \%$ overlap); and the precentral, central, and postcentral gyri ( $\sim 30 \%$ overlap). The lesion volume was significantly different across groups $\left(F_{2,22}=13.68, p \leq 0.001\right)$, 

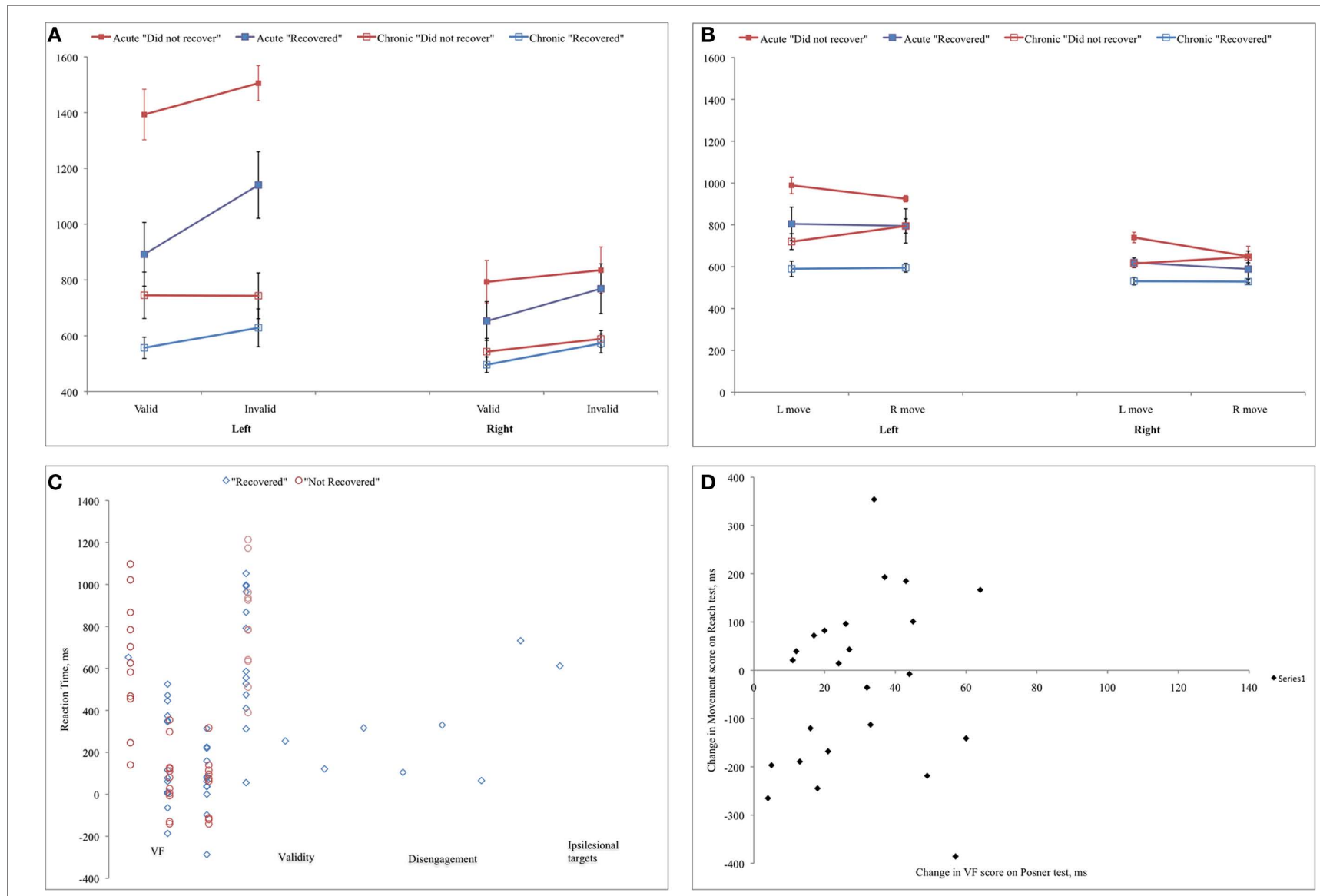

FIGURE 5 | (A,B) Performance on Posner and Reach tasks for patients who showed good vs. poor recovery on clinical neglect tests at the chronic stage; (C) Scatter plot of individual subjects' effects on Posner task at the acute stage for patients who showed good vs. poor recovery on clinical neglect tests; (D) Correlation between recovery on Posner and Reach tasks.

with larger average lesion volumes in the TPJ and VFC than subcortical groups $(p \leq 0.001)$. The lesion volumes in the TPJ and VFC groups were not significantly different.

Performance of the three subgroups on the various clinical tests varied considerably by test, as shown in Figure 8, possibly due to very low Ns. Two TPJ subjects had missing data for one or more of the clinical tests; data are only reported for tests with complete information from each group.

\section{Posner task}

On the Posner task, the VFC group performed significantly worse than the other groups (Figure 9). The VFC group was slower overall (Group: $F_{2,16}=6.08, p=0.010$; VFC vs. subcortical, $p=0.010$; VFC vs. TPJ, $p=0.009)$ and was more impaired in reorienting to unattended locations (Validity $\times$ Group $\left(F_{2,16}=3.97\right.$, $p=0.04)$; post hoc: VFC vs. subcortical, $p=0.003$; VFC vs. TPJ, $p=0.032)$. No significant group differences were found for Visual Field or disengagement (VF $\times$ Validity $\times$ Group) effects. The enhanced detection and reorienting deficits in the VFC group recovered over time (Stage $\times$ Group: $F_{2,16}=7.53, p=0.008$; Stage $\times$ Validity $\times$ Group: $F_{2,16}=11.04, p=0.001$; post hoc: VFC vs. subcortical, $p=0.001$; VFC vs. TPJ $p=0.009$ ) and were not observed at the chronic stage.

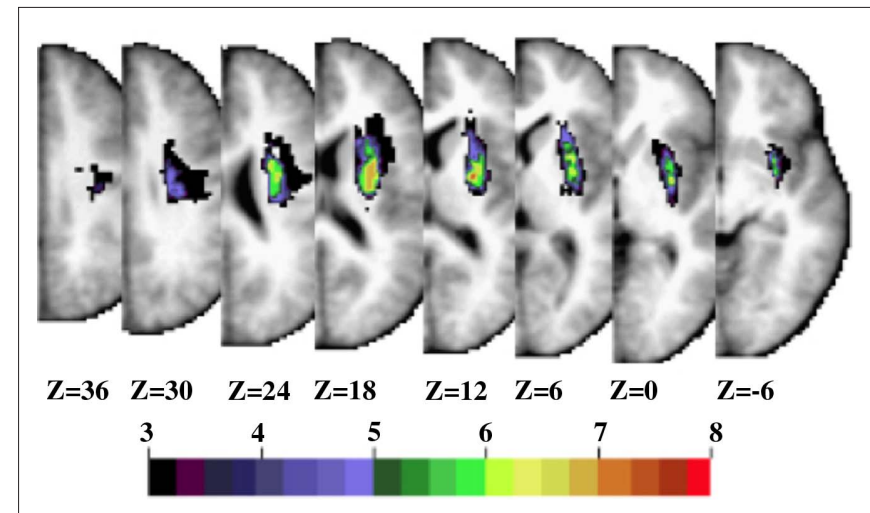

FIGURE 6 | Subcortical group. Horizontal slices of anatomical MRI standardized in Talairach atlas showing the lesion distribution for the 13 patients in the subcortical group. The color scale represents the number of patients with damage in a specific voxel.

When compared to healthy age-matched control subjects, each lesion group showed significant deficits at the acute stage of detection and reorienting, especially in the left visual field (Subcortical vs. Controls: VF $\times$ Group $p<0.001$; Validity $\times$ Group, $p=0.001$; $\mathrm{VF} \times$ Validity $\times$ Group, $p=0.001$; VFC vs. Controls: VF $\times$ Group, 

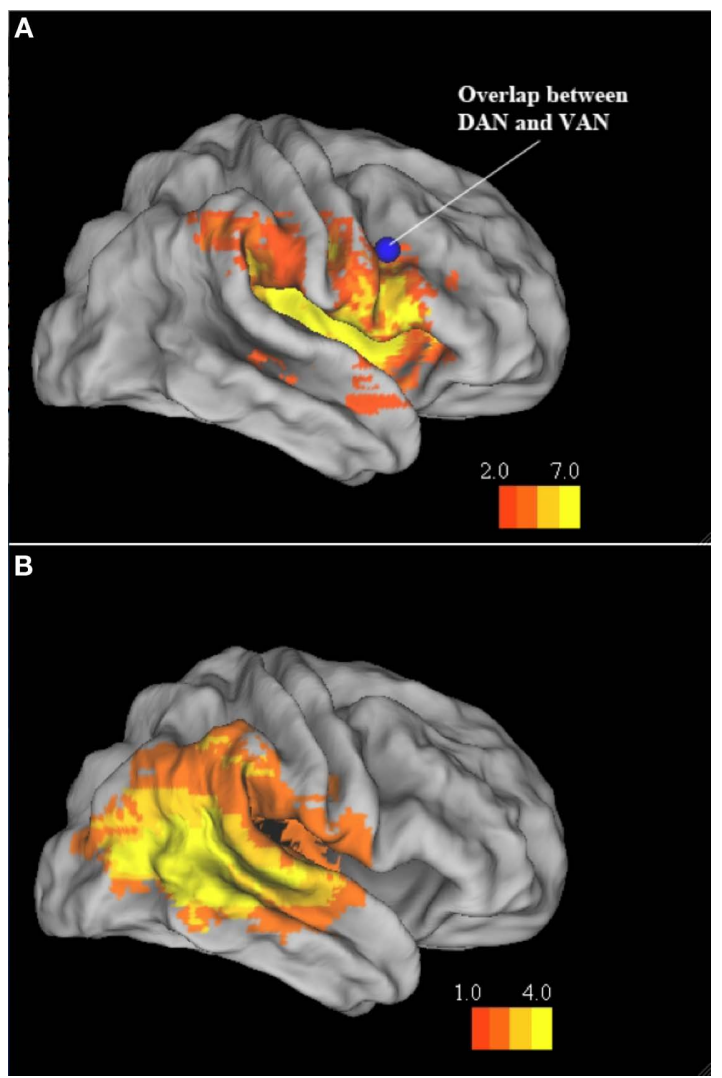

FIGURE 7 | Ventral frontal cortex andTPJ groups. Inflated brain atlas (Caret, PALS) showing regions of maximal cortical damage in the VFC and TPJ subgroups. (A) VFC Group. Blue focus shows the region of overlap found for the dorsal and attention networks by He et al. (2007). (B) TPJ group.

$p<0.001$; Validity $\times$ Group, $p<0.001 ; \mathrm{VF} \times$ Validity $\times$ Group, $p<0.001$; TPJ vs. Controls: VF $\times$ Group, $p<0.001$; Validity $\times$ Group, $p=0.002 ; \mathrm{VF} \times$ Validity $\times$ Group, $p=0.010)$. At the chronic stage deficits in contralateral detection and reorienting were still significant in each group (Subcortical vs. Controls: VF $\times$ Group, $p=<0.001$; Validity $\times$ Group, $p<0.001$; VFC vs. Controls: VF $\times$ Group, $p<0.001$, Validity $\times$ Group, $p=0.005$; TPJ vs. Controls: VF $\times$ Group, $p<0.001$, Validity $\times$ Group, $p=0.001)$, but a disengagement deficit was not present (no significant differences by Group for VF $\times$ Validity). Therefore, patients with VFC damage were especially impaired in detection and reorienting as compared to patients with subcortical and TPJ damage, but detection, reorienting, and disengagement deficits were present in all lesion groups as compared to controls.

\section{Reach task}

In the Reach task, there were no differences among lesion groups (VFC, Subcortical, TPJ) in terms of either RT or misses (Figure 10). Significant motor deficits for initiating contralateral movements were present, as compared to controls, in the TPJ and subcortical groups at the acute stage (Acute RTs: TPJ vs. Control, Direction $\times$ Group: $F_{2,20}=5.37, p=0.027$; Subcortical vs. Control, Direction $\times$ Group: $\left.F_{2,20}=7.33, p=0.01\right)$. In the acute TPJ group only, a motor deficit for initiating contralateral movements was also significantly present (Movement $\times \mathrm{VF} \times$ Group: $F_{2,20}=6.77$, $p=0.014)$. Neither the Subcortical nor the VFC group showed significant directional deficits as compared to controls.

\section{DISCUSSION \\ RELATIVE IMPORTANCE OF DIFFERENT SPATIAL DEFICITS AND THEIR RECOVERY}

This study examined the relative strength of different lateralized processing deficits in spatial neglect and their recovery over time. We found that lateralized perceptual and directional motor impairments were dissociated and followed a different pattern of recovery.

Impairments in lateralized perception were strongly present in our acute spatial neglect group, while directional motor impairments were largely absent. Importantly for the clinician, these lateralized perceptual deficits appeared especially amenable to recovery. One caveat is that the enrollment criteria may have biased the results by favoring the selection of patients with perceptual/ attention impairments over motor deficits, although, this is unlikely since patients were enrolled based the presence of neglect along a number of dimensions (visual, auditory, tactile, motor, personal, non-personal). For example, in our total sample about 95\% were classified as having neglect based on ADL. Another possibility is that the Reach task was less sensitive than the original Mattingley et al. (1998) task, as our task required diagonal rather than purely ipsi- or contralesional horizontal movements. Therefore, it is possible that the relative deficits identified by the two tests were attributable to specific characteristics of the tests rather than underlying deficits.

Although only a handful of studies have longitudinally measured processing deficits in neglect, their results are consistent with the conclusion that measurements of lateralized perceptual deficits in neglect patients are diagnostic and robust. Morrow and Ratcliff (1988) showed that the disengagement deficit in the Posner task (Posner et al., 1984) correlated with severity of neglect, as measured by pencil-and-paper cancelation tasks, at 6-8 weeks, and longitudinally in four patients that were followed at a more chronic stage (4-6 months; but see for a negative correlation Sacher et al., 2004). More recently Farne et al. (2004) studied a group of 33 right brain damaged patients, of whom 23 had spatial neglect, longitudinally from within 2 months after injury. They used a large battery of pencil-and-paper and computerized tasks designed to test spatial attention and vigilance as well as other neglect-related deficits. Their findings are consistent with ours, showing left side perceptual/attention deficits, some recovery over time, but persistence at the chronic stage. As our patients were tested at a later time point than those in Farne et al. (2004; $35 \pm 5$ weeks), these spatial deficits may persist permanently.

The strong recovery of perceptual/attention deficits has been associated in two recent neuroimaging studies to the restoration of task-driven and spontaneous activity in dorsal parietal and frontal areas that are active for shifts of spatial attention, visual selection, and eye movements but are typically not damaged by lesions causing neglect (Corbetta et al., 2005; He et al., 2007). At the acute stage, task-evoked responses in right hemisphere dorsal fronto-parietal regions are considerably reduced while some regions in the left hemisphere show an enhanced response. Over the course of recovery, a more normal balance of responses in two 


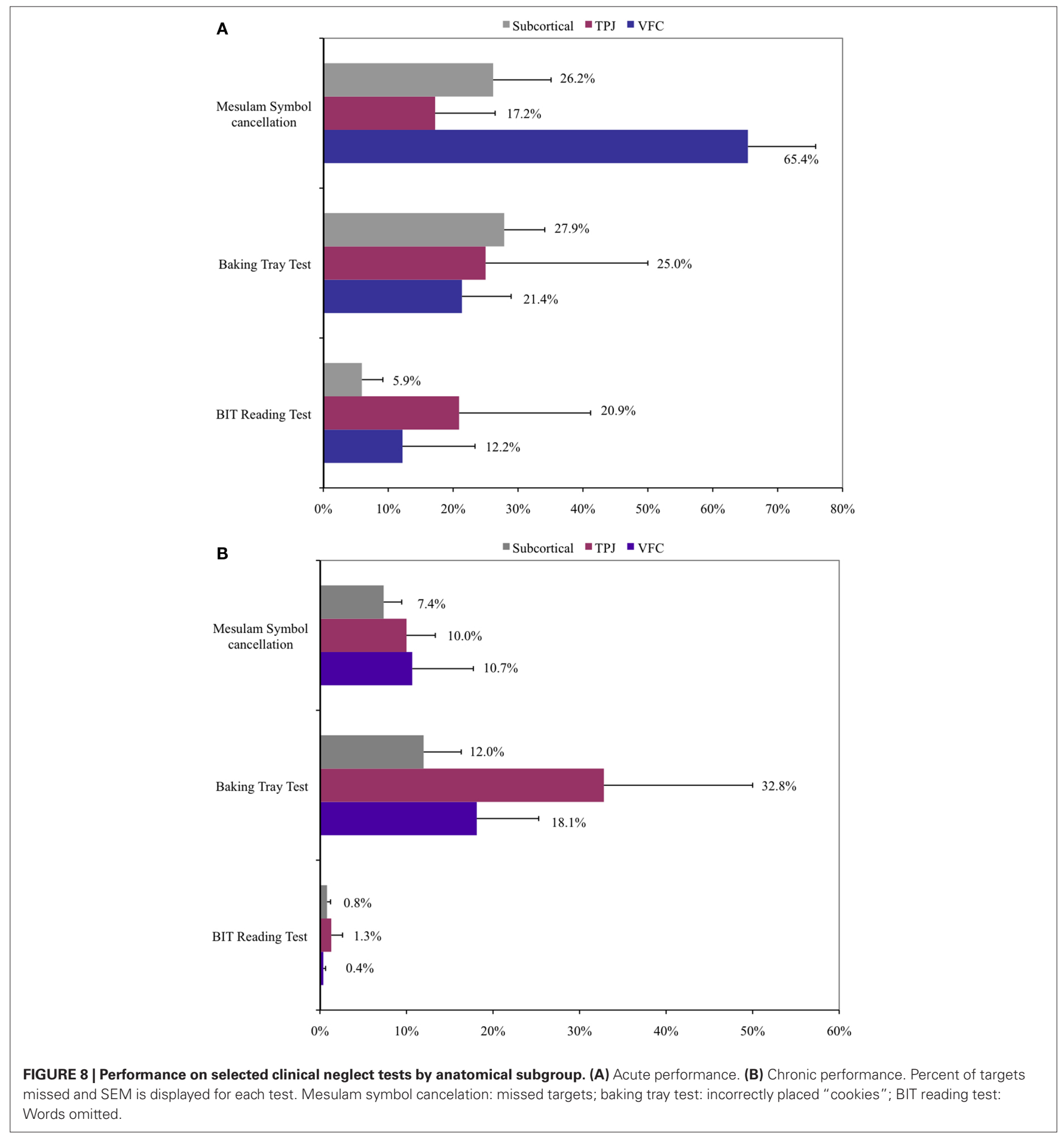

hemispheres is restored (Corbetta et al., 2005). Similarly, at the acute stage, functional connectivity, i.e., temporal correlation of spontaneous activity, between dorsal fronto-parietal regions in left and right hemispheres is reduced and the reduction correlates with the severity of the lateralized deficits observed in the Posner task (He et al., 2007), but the connectivity recovers over time. Functional recovery can also occur in ventral temporo-parietal and frontal regions, and is associated with improvement of attentional functions like reorienting (Corbetta et al., 2005). Restoration of cortical metabolism in ventral anterior and posterior cortex has been also reported after subcortical and basal ganglia damage (Hillis et al., 2005; Karnath et al., 2005).

A final notable finding was that in our neglect group, directional motor problems were mild and did not change significantly over the course of recovery. One study has reported that directional hypokinesia is most associated with lesions to the putamen and surrounding white 


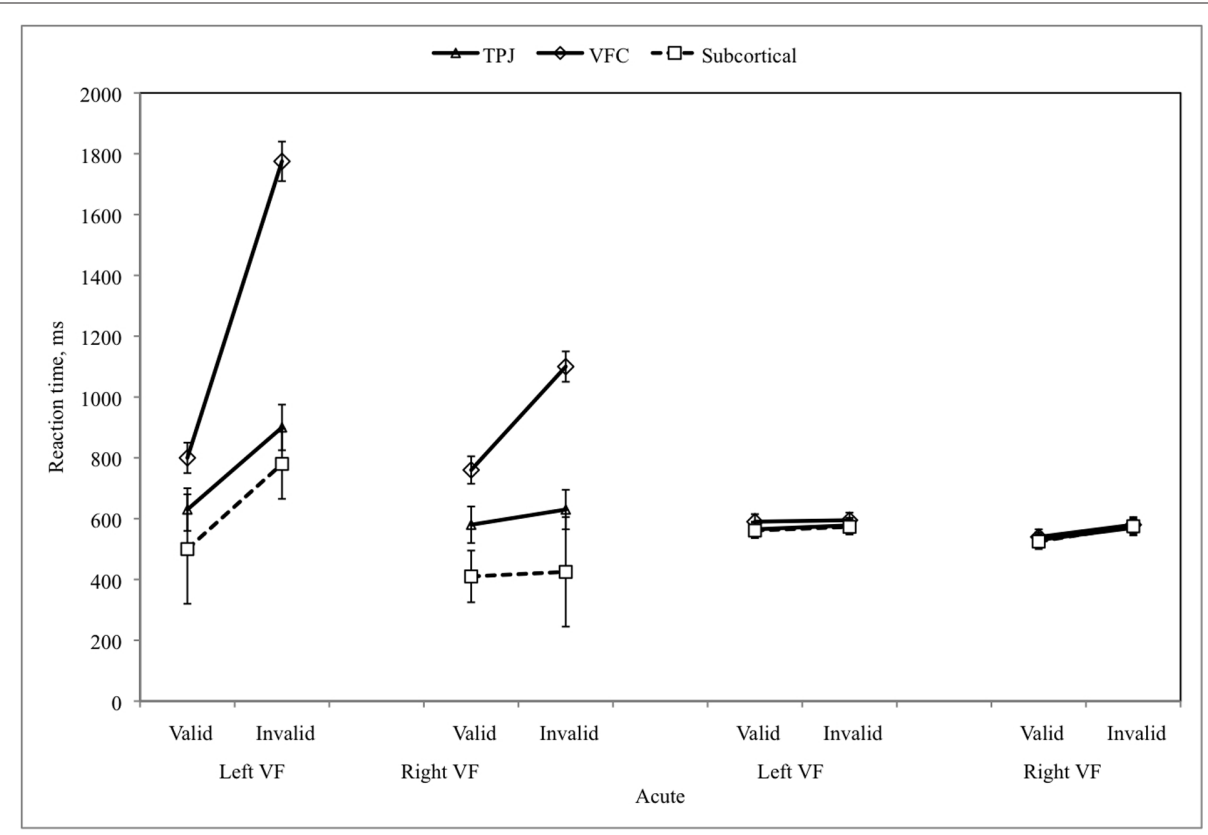

FIGURE 9 | Subgroup analysis of Posner task, reaction time (substituting 2 s for miss trials).

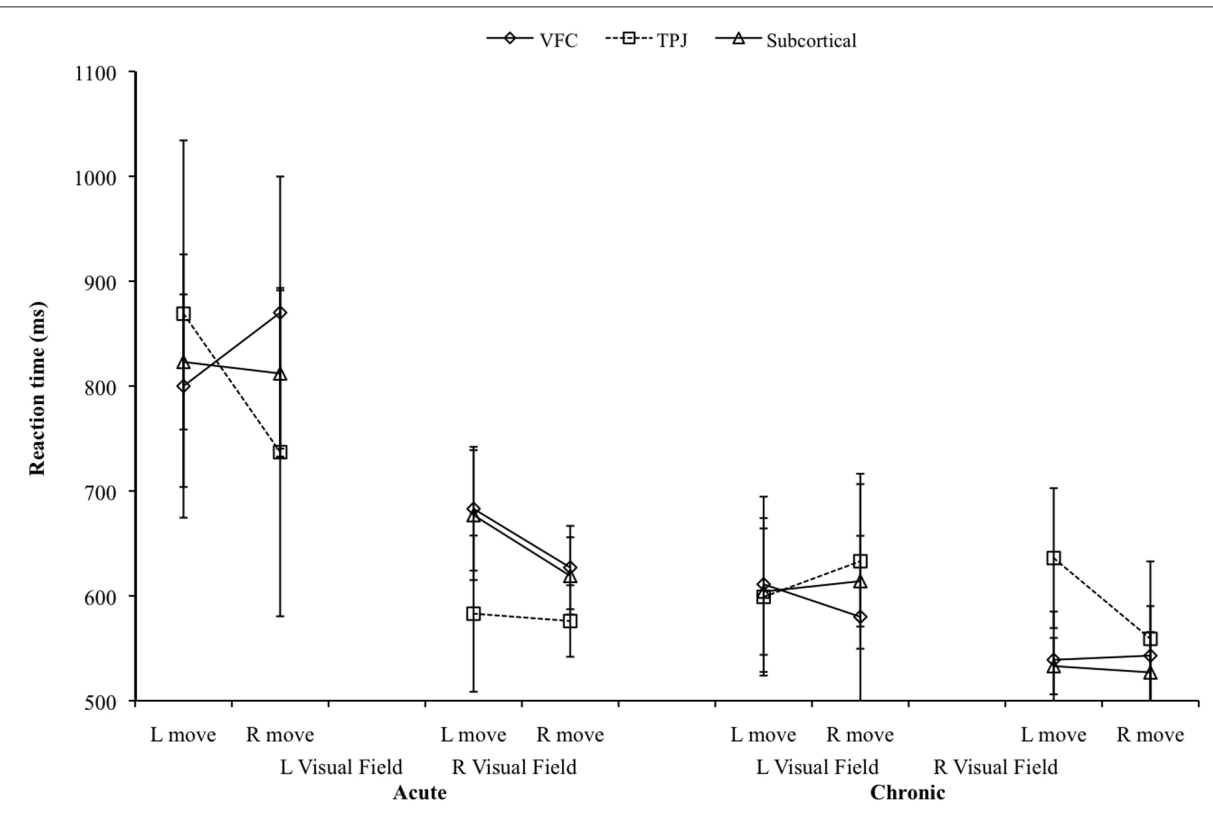

FIGURE 10 | Subgroup analysis of Reach task, reaction times.

matter (Sapir et al., 2007). The poor recovery of directional hypokinesia in neglect patients may reflect the fact that these regions are often structurally damaged by lesions that cause neglect, unlike the dorsal frontoparietal regions thought to underlie lateralized perceptual deficits.

\section{FRONTAL CORTEX AS A SITE OF CONVERGENCE FOR SPATIAL AND NON-SPATIAL DEFICITS}

Patients with lesions involving VFC showed the strongest detection and reorienting deficits, as compared to TPJ and subcortical groups, even though the average lesion volume in the VFC group was not larger than the other groups (actually smaller than TPJ). VFC lesions involved damage in multiple cortical regions including inferior frontal gyrus, anterior insula, and the white matter underlying ventral and middle frontal cortex. In some patients the lesion extended dorsally in the precentral gyrus and posteriorly in the parietal operculum and postcentral cortex.

Previous studies had proposed a critical association between the disengagement deficit, i.e., reorienting specifically to the contralesional field, and damage of the STG, part of TPJ (Friedrich et al., 1998; Ellison et al., 2004; Karnath et al., 2004; 
Corbetta et al., 2005; Gharabaghi et al., 2006). We found that VFC lesions had a more pronounced overall effect than TPJ lesions on reorienting attention, although the disengagement effect did not significantly differ between groups. This result is consistent with findings that other sites may be equally or more likely than STG to produce neglect, including inferior parietal cortex (IPL; Mort et al., 2003; Golay et al., 2008). Farne et al. (2004) examined the relationship between anatomy and neglect recovery, and found no critical relationship between STG and neglect at either the acute or chronic stages, or with recovery of neglect, while other parietal and frontal areas were critical for neglect in their sample.

While it is clear that no one site is exclusively responsible for the heterogeneous neglect syndrome, an interesting emerging notion is that VFC is a site of convergence of spatial and non-spatial functions, both of which are damaged in neglect patients. From this perspective, it was interesting that the VFC group showed pronounced detection and reorienting deficits in both the contralesional and ipsilesional fields. VFC may participate in functional communication between frontal and parietal cortices, with neglect arising as a result of a disconnection in this communication (Bartolomeo et al., 2007). Recent functional connectivity studies of the dorsal and ventral attention networks show that these two networks represent two separate and largely independent functional systems that fluctuate in the awake resting state (Fox et al., 2006). The only region in the brain with shared synchrony between the two systems is the right prefrontal cortex that includes inferior and middle frontal gyri (He et al., 2007). This observation suggests that the two attention networks may interact through the right prefrontal cortex, an idea consistent with recent findings that the lateral prefrontal cortex is an important site of convergence for stimulus-driven and goal-directed attention (Asplund et al., 2010). Interestingly, the VFC lesions in the current study included the right prefrontal region in question (see Figure 6). Therefore, damage to white matter connections between frontal and parietal cortex may cause large imbalances in posterior parietal cortex due to impaired functional connectivity between dorsal and ventral fronto-parietal networks (Thiebaut de Schotten

\section{REFERENCES}

Appelros, P., Karlsson, G. M., Seiger, A., and Nydevik, I. (2002). Neglect and anosognosia after first-ever stroke: incidence and relationship to disability. J. Rehabil. Med. 34, 215-220.

Asplund, C. L., Todd, J. J., Snyder, A. P., and Marois, R. (2010). A central role for the lateral prefrontal cortex in goal-directed and stimulus-driven attention. Nat. Neurosci. 13, 507-512.

Astafiev, S. V., Shulman, G. L., Stanley, C. M., Snyder, A. Z., Van Essen, D. C., and Corbetta, M. (2003). Functional organization of human intraparietal and frontal cortex for attending, looking, and pointing. J. Neurosci. 23, 4689-4699.

Azouvi, P., Olivier, S., de Montety, G., Samuel, C., Louis-Dreyfus, A., and Tesio, L. (2003). Behavioral assessment of unilateral neglect: study of the psychometric properties of the

et al., 2005), hence explaining the severe spatial deficits in the VFC group. This hypothesis was supported in a study of BOLD functional connectivity in neglect patients, which reported that the degree of impaired synchrony between TPJ and prefrontal cortex was strongly correlated with the degree of impaired synchrony in dorsal parietal cortex (He et al., 2007). Furthermore, patients with severe neglect and more impaired interregional synchrony had lesions in the white matter corresponding to the arcuate and superior longitudinal fasciculus, which respectively connect TPJ to dorsolateral prefrontal cortex, and the latter with dorsal parietal cortex (He et al., 2007). Overall, then neglect can be thought of a syndrome in which damage to ventral frontal and temporo-parietal cortex causes not only behavioral deficits related to the structural damage proper, but also "functional" deficits related to the disconnection of more dorsal attention areas specialized in directing covert and overt (eye movements) attention to spatial locations (Corbetta and Shulman, 2011).

It should be noted because the current study did not include a control group of right hemisphere-lesioned patients without neglect, any findings regarding lesion overlap may be attributable to the susceptibility of that region to stroke due to vascular distributions. Future studies that included this control group would be useful for clarifying the role of the VFC in producing neglect.

Spatial neglect is a heterogeneous behavioral syndrome. The present results indicate the overwhelming clinical importance of lateralized spatial deficits of attention (orienting, reorienting) and perception, as compared to disturbances of action. Perceptualattention deficits are not only the most severe, but also show the most variability in the course of recovery. Therefore, they should be prime candidates for rehabilitation interventions. Spatial neglect after VFC damage is especially severe and likely reflects impaired fronto-parietal communication.

\section{ACKNOWLEDGMENTS}

We thank Michelle Kincade for data collection, and Ayelet Sapir for data analysis. This work was supported by the National Institute of Mental Health (R01 MH71920-06 to Maurizio Corbetta), and the James S. McDonnell Foundation (grant 99649900004).

Buxbaum, L. J., Ferraro, M. K., Veramonti, T., Farne, A., Whyte, J., Ladavas, E., Frassinetti, F., and Coslett, H. B. (2004). Hemispatial neglect: subtypes, neuroanatomy, and disability. Neurology 62, 749-756.

Cherney, L. R., Halper, A. S., Kwasnica, C. M., Harvey, R. L., and Zhang, M. (2001). Recovery of functional status after right hemisphere stroke: relationship with unilateral neglect. Arch. Phys. Med. Rehabil. 82, 322-328.

Corbetta, M., Kincade, J. M., Ollinger, J. M., McAvoy, M. P., and Shulman, G. L. (2000). Voluntary orienting is dissociated from target detection in human posterior parietal cortex. Nat. Neurosci. 3, 292-297.

Corbetta, M., Kincade, M. J., Lewis, C., Snyder, A. Z., and Sapir, A. (2005). Neural basis and recovery of spatial attention deficits in spatial neglect. Nat. Neurosci. 8, 1603-1610.
Corbetta, M., Patel, G., and Shulman, G. L. (2008). The reorienting system of the human brain: from environment to theory of mind. Neuron 58, 306-324. Corbetta, M., and Shulman, G. L. (2002). Control of goal-directed and stimulusdriven attention in the brain. Nat. Rev. Neurosci. 3, 201-215.

Corbetta, M., and Shulman, G. L. (2011). Spatial neglect and attention networks. Annu. Rev. Neurosci. doi: 10.1146/ annurev-neuro-061010-113731. [Epub ahead of print].

Ellison, A., Schindler, I., Pattison, L., and Milner, D. (2004). An exploration of the role of the superior temporal gyrus in visual search and spatial perception using TMS. Brain 127, 2307-2315.

Farne, A., Buxbaum, L. J., Ferraro, M., Frassinetti, F., Whyte, J., Veramonti, T., Angeli, V., Coslett, H. B., and Ladavas, E. (2004). Patterns of spontaneous recovery of neglect and 
associated disorders in acute right brain-damaged patients. J. Neurol. Neurosurg. Psychiatr. 75, 1401-1410.

Fox, M. D., Corbetta, M., Snyder, A. Z., Vincent, J. L., and Raichle, M. E. (2006).Spontaneous neuronal activity distinguishes human dorsal and ventral attention systems. Proc. Natl. Acad. Sci. U.S.A. 2006 103, 10046-10051.

Friedrich, F. J., Egly, R., Rafal, R. D., and Beck, D. (1998). Spatial attention deficits in humans: a comparison of superior parietal and temporal-parietal junction lesions. Neuropsychology 12, 193-207.

Gharabaghi, A., Fruhmann, B. M., Tatagiba, M., and Karnath, H. O. (2006). The role of the right superior temporal gyrus in visual search insights from intraoperative electrical stimulation. Neuropsychologia 45, 465.

Golay, L., Schnider, A., and Ptak, R. (2008). Cortical and subcortical anatomy of chronic spatial neglect following vascular damage. Behav. Brain Funct. 4,43.

Halligan, P. W., Cockburn, J., and Wilson, B. A. (1991). The behavioural assessment of visual neglect. Neuropsychol. Rehabil. 1, 5-32.

Halligan, P. W., Fink, G. R., Marshall J. C., and Vallar, G. (2003). Spatial cognition: evidence from visual neglect. Trends Cogn. Sci. 7, 125-133.

He, B. J., Snyder, A. Z., Vincent, J. L., Epstein, A., Shulman, G. L., and Corbetta, M. (2007). Breakdown of intrinsic brain synchrony in spatial neglect: a novel mechanism to explain brain-behavior relationships after stroke. Neuron 53, 905-918.

Heilman, K. M., Bowers, D., Coslett, H. B., Whelan, H., and Watson R. T. (1985). Directional hypokinesia: prolonged reaction times for leftward movements in patients with right hemisphere lesions and neglect. Neurology 35, 855-859.

Heilman, K. M., Bowers, D., Valenstein, E., and Watson, R. T. (1987) "Hemispace and hemispatial neglect," in Neurophysiological and Neuropsychological Aspects of Spatial Neglect, ed. M. Jeannerod (North
Holland: Elsevier Science Publishers), pp. 115-150.

Hillis, A. E., Newhart, M., Heidler, J., Barker, P. B., Herskovits, E. H., and Degaonkar, M. (2005). Anatomy of spatial attention: insights from perfusion imaging and hemispatial neglect in acute stroke. J. Neurosci. 25, 3161-3167.

Husain, M., Mattingley, J. B., Rorden C., Kennard, C., and Driver, J. (2000). Distinguishing sensory and motor biases in parietal and frontal neglect. Brain 123, 1643-1659.

Karnath, H. O., Fruhmann, B., Kuker, W., and Rorden, C. (2004). The anatomy of spatial neglect based on voxelwise statistical analysis: a study of 140 patients. Cereb. Cortex 14, 1164-1172.

Karnath, H. O., Rorden, C., and Ticini, L. F. (2005). Normalized perfusion MRI to identify common areas of dysfunction: patients with basal ganglia neglect. Brain 128, 2462-2469.

Katz, N., Hartman-Maeir, A., Ring, H., and Soroker, N. (1999). Functional disability and rehabilitation outcome in right hemisphere damaged patients with and without unilateral spatial neglect. Arch. Phys. Med. Rehabil. 80, 379-384.

Katzman, R., Brown, T., Fuld, P., Peck, A., Schechter, R., and Schimmel, H. (1983). Validation of a short orientation-memory-concentration test of cognitive impairment. Am. J. Psychiatry 140, 734-739.

Kincade, J. M., Abrams, R. A., Astafiev, S. V., Shulman, G. L., and Corbetta, M. (2005). An event-related functional magnetic resonance imaging study of voluntary and stimulus-driven orienting of attention. J. Neurosci. 25 , 4593-4604.

Mattingley, J. B., Husain, M., Rorden, C., Kennard, C., and Driver, J. (1998). Motor role of human inferior parietal lobe revealed in unilateral neglect patients. Nature 392, 179-182.

Mesulam, M. M. (1985). "Mental state assessment of young and elderly adults in behavioral neurology," in
Principles of Behavioral Neurology, ed. M. Mesulam (Philadelphia: F. A. Davis Company), 73-121.

Mesulam, M. M. (1999). Spatial attention and neglect: parietal, frontal and cingulate contributions to the mental representation and attentional targeting of salient extrapersonal events. Philos. Trans. R. Soc. Lond., B, Biol. Sci. 354 1325-1346.

Morrow, L. A., and Ratcliff, G. (1988). The disengagement of covert attention and the neglect syndrome. Psychobiology $16,261-269$.

Mort, D. J., Malhotra, P., Mannan, S. K. Rorden, C., Pambakian, A., Kennard, C., and Husain, M. (2003). The anatomy of visual neglect. Brain 126 1986-1997.

Paolucci, S., Grasso, M. G., Antonucci, G., Bragoni, M., Troisi, E., Morelli, D., Coiro, P., De Angelis, D., and Rizzi, F. (2001). Mobility status after inpatient stroke rehabilitation: 1-year follow-up and prognostic factors. Arch. Phys. Med. Rehabil. 82, 2-8.

Pedersen,P.M., Jorgensen,H.S., Nakayama, H., Raaschou, H. O., and Olsen T. S. (1997). Hemineglect in acute stroke: incidence and prognostic implications. The Copenhagen Stroke Study. Am. J. Phys. Med. Rehabil. 76, 122-127.

Posner, M. I. (1980). Orienting of attention. Q. J. Exp. Psychol. 32, 3-25.

Posner, M. I., Walker, J. A., Friedrich, F. J., and Rafal, R. D. (1984). Effects of parietal injury on covert orienting of attention. J. Neurosci. 4, 1863-1874.

Rastelli, F., Funes, M.-J., Lupiáñez, J., Duret, C., and Bartolomeo, P. (2008). Left visual neglect: is the disengage deficit space- or object-based? Exp. Brain Res. 187, 439-446.

Sacher,Y., Serfaty, C., Deouell, L., Sapir,A., Henik, A., and Soroker, N. (2004). Role of disengagement failure and attentional gradient in unilateral spatial neglect: a longitudinal study. Disabil. Rehabil. 26, 746-755.

Sapir,A., Kaplan, J., He, B. J., and Corbetta, M. (2007). Anatomical correlates of directional hypokinesia in patients with hemispatial neglect. J. Neurosci. 27, 4045-4051.

Sheline, Y. I., Price, J. L., Vaishnavi, S. N., Mintun, M.A., Barch, D. M., Epstein,A. A.,Wilkins, C.H.,Snyder,A.Z., Couture, L., Schechtman, K., and McKinstry, R. C. (2008). Regional white matter hyperintensity burden in automated segmentation distinguishes late-life depressed subjects from comparison subjects matched for vascular risk factors. $A m$. J. Psychiatry 165, 524-532.

Stone, S. P., Halligan, P. W., and Greenwood, R. J. (1993). The incidence of neglect phenomena and related disorders in patients with an acute right or left hemisphere stroke. Age Ageing 22, 46-52.

Tham, K., and Tegner, R. (1996). The baking tray task: a test of spatial neglect. Neuropsychol. Rehabil. 6, 19-25.

Thiebaut de Schotten, M., Urbanski, M., Duffau, H., Volle, E., Lévy, R., Dubois, B., and Bartolomeo, P. (2005). Direct evidence for a parietal-frontal pathway subserving spatial awareness in humans. Science 309, 2226-2228.

Wilson, B., Cockburn, J., and Halligan, P. (1987). Development of a behavioral test of visuospatial neglect. Arch. Phys. Med. Rehabil. 68, 98-102.

Conflict of Interest Statement: The authors declare that the research was conducted in the absence of any commercial or financial relationships that could be construed as a potential conflict of interest.

Received: 20 July 2010; accepted: 07 March 2011; published online: 04 April 2011.

Citation: Rengachary J, He BJ, Shulman GL and Corbetta M (2011) A behavioral analysis of spatial neglect and its recovery after stroke. Front. Hum. Neurosci. 5:29. doi: 10.3389/fnhum.2011.00029

Copyright $\odot 2011$ Rengachary, He, Shulman and Corbetta. This is an open-access article subject to a non-exclusive license between the authors and Frontiers Media SA, which permits use, distribution and reproduction in other forums, provided the original authors and source are credited and other Frontiers conditions are complied with. 